\title{
Low Energy Thresholds and the Renormalization Group in the MSSM
}

\author{
A. B. Lahanas $\dagger$ \\ University of Athens, Physics Department, Nuclear and Particle Physics Section \\ Ilissia, GR - 15771 Athens, GREECE \\ and \\ K. Tamvakis $\ddagger$ \\ Division of Theoretical Physics, University of Ioannina \\ Ioannina, GR - 45110 GREECE
}

\begin{abstract}
We derive the 1-loop Renormalization Group Equations for the parameters of the Minimal Supersymmetric Standard Model (MSSM) taking into account the successive decoupling of each sparticle below its threshold. This is realized by a step function at the level of each graph contributing to the Renormalization Group Equations.
\end{abstract}

Dedicated to the memory of Spyros Vlassopulos, colleague and friend

IOA-314/94

UA/NPPS - 16/1994

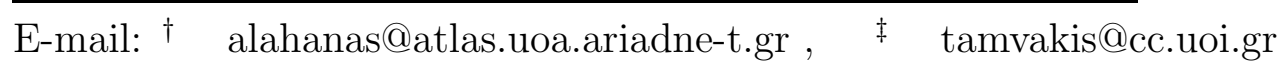


The softly broken version of the Minimal Supersymmetric Standard Model (MSSM) 迎 is well known to lead to Electroweak Symmetry breaking through radiative corrections 2 The study of radiative corrections is most conveniently done by the use of the Renormalization Group Equations (RGE's) for the running parameters of the model. Ultimately these energy dependent parameters should be related to the physical ones. One popular way to realize this program 3 exclusively in the framework of the (RGE's) is the following:

We derive the Renormalization Group Equations for running masses and couplings in the $\overline{D R}$ scheme. As we come down in energy and encounter the heaviest particle threshold we switch to another "effective" theory from which the heavy particle is removed and so on. Although the $\overline{D R}$ scheme is mass independent, we enforce the Decoupling Theorem at the level of the (RGE's) by replacing the full theory by a succession of effective theories. For a particular running mass $m(Q)$ this treatment of its Renormalization Group Equation will stop when we encounter the corresponding physical mass determined by the condition $m\left(m_{p h}\right)=m_{p h}$. Both the step function approximation on the RGE's and this last condition for the physical mass are approximations that keep the leading logarithmic part in 2-point functions and ignore the constant part. The popularity of this scheme is based on the fact that one stays only within the Renormalization Group Equations 6 and does not have to consider the finite parts of the Green functions.

In the present paper we derive in the $\overline{D R}$ scheme the 1-loop RGE's for both dimensionless (gauge and Yukawa couplings) and dimensionful parameters (soft masses and cubic couplings). At the level of each graph we enforce decoupling by inserting a theta function $\theta_{m} \equiv \theta\left(Q^{2}-m^{2}\right)$ that counts the contribution of a particle of mass $m$ at energies $Q>m$. In order for such a program to be carried out we should calculate the infinities of the two and three point functions of all particles involved. The use of the superfield formalism in a non-supersymmetric theory, although possible through the utilization of spurious superfields, becomes difficult due to the successive decoupling occurring at the component level. One has to resort to the component formulation of the theory where the non-Renormalization Theorem does not apply. The details of the calculation along with the predictions of the MSSM for the sparticle mass spectrum using the modified RGE's described above will be presented elsewhere 1 .

The parameters of the MSSM are defined by the superpotential (suppressing all indices)

$$
\mathcal{W}=Y_{u} Q H_{2} U^{c}+Y_{d} Q H_{1} D^{c}+Y_{e} L H_{1} E^{c}+\mu H_{1} H_{2}
$$

and the soft supersymmetry-breaking interaction Lagrangian

$$
\begin{aligned}
-\mathcal{L}_{\text {soft }} & =\sum_{i} m_{i}^{2}\left|\Phi_{i}\right|^{2}+\left(Y_{u} A_{u} \tilde{Q} H_{2} \tilde{U}^{c}+Y_{d} A_{d} \tilde{Q} H_{1} \tilde{D}^{c}+Y_{e} A_{e} \tilde{L} H_{1} \tilde{E}^{c}+\text { h.c. }\right) \\
& +\left(\mu B H_{1} H_{2}+\text { h.c. }\right)+\frac{1}{2} \sum_{a} M_{a} \bar{\lambda}_{a} \lambda_{a} .
\end{aligned}
$$

The beta functions for the gauge and Yukawa couplings are, with $t \equiv \log \left(\frac{Q^{2}}{M_{G U T}^{2}}\right)$ and keeping only the Yukawa couplings $Y_{t, b, \tau}$ of the third generation fermions, 


$$
\begin{aligned}
& \frac{d g_{i}}{d t} \equiv \beta\left(g_{i}\right)=\frac{b_{i}}{2(4 \pi)^{2}} T_{i} g_{i}{ }^{3}, \quad i=1,2,3 \\
& b_{1,2,3}=\frac{33}{5}, 1,-3 \\
& \frac{d Y_{\tau}}{d t} \equiv \beta\left(Y_{\tau}\right)=\frac{Y_{\tau}}{(4 \pi)^{2}}\left\{-\frac{3}{2} T_{\tau 2} g_{2}{ }^{2}-\frac{9}{10} T_{\tau 1} g_{1}^{2}+2 T_{\tau \tau} Y_{\tau}^{2}+\frac{3}{2} Y_{b}^{2}\right\} \\
& \frac{d Y_{b}}{d t} \equiv \beta\left(Y_{b}\right)= \\
& \quad \frac{Y_{b}}{(4 \pi)^{2}}\left\{-\frac{8}{3} T_{b 3} g_{3}^{2}-\frac{3}{2} T_{b 2} g_{2}{ }^{2}-\frac{7}{30} T_{b 1} g_{1}{ }^{2}+\frac{1}{2} T_{b t} Y_{t}^{2}+3 T_{b b} Y_{b}^{2}+\frac{1}{2} Y_{\tau}^{2}\right\} \\
& \frac{d Y_{t}}{d t} \equiv \beta\left(Y_{t}\right)=\frac{Y_{t}}{(4 \pi)^{2}}\left\{-\frac{8}{3} T_{t 3} g_{3}{ }^{2}-\frac{3}{2} T_{t 2} g_{2}{ }^{2}-\frac{13}{30} T_{t 1} g_{1}{ }^{2}+3 T_{t t} Y_{t}^{2}+\frac{1}{2} T_{t b} Y_{b}^{2}\right\}
\end{aligned}
$$

The threshold coefficients $T_{i}, T_{e i}$, etc appearing in the expressions above are shown in table I.

The beta functions for the cubic couplings are円

$$
\begin{aligned}
\frac{d A_{\tau}}{d t} & =\frac{1}{(4 \pi)^{2}}\left\{-3 g_{2}{ }^{2} M_{2} \theta_{\tilde{W} \tilde{H}_{1}}-\frac{3}{5} g_{1}^{2} M_{1}\left(2+\theta_{\tilde{H}_{1}}\right) \theta_{\tilde{B}}\right. \\
& \left.+3 Y_{b}^{2} A_{b} \theta_{\tilde{D} \tilde{Q}}+4 Y_{\tau}^{2} A_{\tau}+A_{\tau}\left[Z_{\tau 1} g_{1}^{2}+Z_{\tau 2} g_{2}^{2}+Z_{\tau \tau} Y_{\tau}^{2}\right]\right\} \\
\frac{d A_{b}}{d t} & =\frac{1}{(4 \pi)^{2}}\left\{-\frac{16}{3} g_{3}{ }^{2} M_{3} \theta_{\tilde{G}}-3 g_{2}^{2} M_{2} \theta_{\tilde{W} \tilde{H}_{1}}-\frac{1}{30} g_{1}^{2} M_{1}\left(-4+18 \theta_{\tilde{H}_{1}}\right) \theta_{\tilde{B}}\right. \\
& +Y_{\tau}^{2} A_{\tau} \theta_{\tilde{E} \tilde{L}}+Y_{t}^{2} A_{t} \theta_{H_{2} \tilde{U}}+6 Y_{b}^{2} A_{b} \\
& \left.+A_{b}\left[Z_{b 3} g_{3}^{2}+Z_{b 2} g_{2}^{2}+Z_{b 1} g_{1}^{2}+Z_{b t} Y_{t}^{2}+Z_{b b} Y_{b}^{2}\right]\right\} \\
\frac{d A_{t}}{d t} & =\frac{1}{(4 \pi)^{2}}\left\{-\frac{16}{3} g_{3}^{2} M_{3} \theta_{\tilde{G}}-3 g_{2}^{2} M_{2} \theta_{\tilde{W} \tilde{H}_{2}}-\frac{1}{15} g_{1}^{2} M_{1}\left(4+9 \theta_{\tilde{H}_{2}}\right) \theta_{\tilde{B}}\right. \\
& +6 Y_{t}^{2} A_{t} \theta_{H_{1} \tilde{U}}+Y_{b}^{2} A_{b} \theta_{H_{1} \tilde{D}} \\
& \left.+A_{t}\left[Z_{t 3} g_{3}{ }^{2}+Z_{t 2} g_{2}^{2}+Z_{t 1} g_{1}^{2}+Z_{t t} Y_{t}^{2}+Z_{t b} Y_{b}^{2}\right]\right\}
\end{aligned}
$$

In our notation $\theta_{a b} \equiv \theta_{a} \theta_{b} \quad \theta_{a b c} \equiv \theta_{a} \theta_{b} \theta_{c}$. In all the expressions throughout this paper we assume that the Yukawa couplings are diagonal in family space. The coefficients $Z_{q i}$

$1 \tilde{G}, \tilde{W}, \tilde{B}$ denote the $S U(3), S U(2)$ and $U(1)$ gauge fermions respectively. $\tilde{H}_{1}, \tilde{H}_{2}$ are Higgs fermions ( Higgsinos ). 
in the expressions for the cubic couplings above vanish above all thresholds and in this case one recovers the well known RGEs .

The beta functions for the scalar masses are given by the following RGEs ${ }^{2}$. The masses $m_{1,2}^{2}$ appearing in the RGEs below refer to the Higgs masses squared which are related to the their soft masses $m_{H_{1}, H_{2}}^{2}$ and the mixing parameter $\mu$ by $m_{1,2}^{2}=m_{H_{1}, H_{2}}^{2}+\mu^{2}$.

$$
\begin{aligned}
& \frac{d m_{\tilde{Q}}^{2}}{d t}=\frac{1}{(4 \pi)^{2}}\left\{-\left[\frac{8}{3} g_{3}{ }^{2}\left(\theta_{\tilde{Q}}-\theta_{\tilde{G}}\right)+\frac{3}{2} g_{2}{ }^{2}\left(\theta_{\tilde{Q}}-\theta_{\tilde{W}}\right)+\frac{1}{30} g_{1}{ }^{2}\left(\theta_{\tilde{Q}}-\theta_{\tilde{B}}\right)\right] m_{\tilde{Q}}^{2}\right. \\
& -\frac{16}{3} g_{3}^{2} M_{3}^{2} \theta_{\tilde{G}}-3 g_{2}{ }^{2} M_{2}^{2} \theta_{\tilde{W}}-\frac{1}{15} g_{1}{ }^{2} M_{1}^{2} \theta_{\tilde{B}}+\frac{1}{10} g_{1}{ }^{2} S \\
& \left.+Y_{t}^{2}\left[m_{\tilde{Q}}^{2} \theta_{\tilde{H}_{2}}+m_{\tilde{U}}^{2} \theta_{\tilde{U}}+m_{2}^{2} \theta_{H_{2}}+A_{t}^{2} \theta_{H_{2} \tilde{U}}+\mu^{2}\left(\theta_{H_{1} \tilde{U}}-2 \theta_{\tilde{H}_{2}}\right)\right]\right\} \\
& \left.+Y_{b}^{2}\left[m_{\tilde{Q}}^{2} \theta_{\tilde{H}_{1}}+m_{\tilde{D}}^{2} \theta_{\tilde{D}}+m_{1}^{2} \theta_{H_{1}}+A_{b}^{2} \theta_{H_{1} \tilde{D}}+\mu^{2}\left(\theta_{H_{2} \tilde{D}}-2 \theta_{\tilde{H}_{1}}\right)\right]\right\} \\
& \frac{d m_{\tilde{U}}^{2}}{d t}=\frac{1}{(4 \pi)^{2}}\left\{-\left[\frac{8}{3} g_{3}^{2}\left(\theta_{\tilde{U}}-\theta_{\tilde{G}}\right)+\frac{8}{15} g_{1}^{2}\left(\theta_{\tilde{U}}-\theta_{\tilde{B}}\right)\right] m_{\tilde{U}}^{2}\right. \\
& -\frac{16}{3} g_{3}{ }^{2} M_{3}^{2} \theta_{\tilde{G}}-\frac{16}{15} g_{1}{ }^{2} M_{1}^{2} \theta_{\tilde{B}}-\frac{2}{5} g_{1}{ }^{2} S \\
& \left.+2 Y_{t}^{2}\left[m_{\tilde{U}}^{2} \theta_{\tilde{H}_{2}}+m_{\tilde{Q}}^{2} \theta_{\tilde{Q}}+m_{2}^{2} \theta_{H_{2}}+A_{t}^{2} \theta_{H_{2} \tilde{Q}}+\mu^{2}\left(\theta_{H_{1} \tilde{Q}}-2 \theta_{\tilde{H}_{2}}\right)\right]\right\} \\
& \frac{d m_{\tilde{D}}^{2}}{d t}=\frac{1}{(4 \pi)^{2}}\left\{-\left[\frac{8}{3} g_{3}^{2}\left(\theta_{\tilde{D}}-\theta_{\tilde{G}}\right)+\frac{2}{15} g_{1}^{2}\left(\theta_{\tilde{D}}-\theta_{\tilde{B}}\right)\right] m_{\tilde{D}}^{2}\right. \\
& -\frac{16}{3} g_{3}{ }^{2} M_{3}^{2} \theta_{\tilde{G}}-\frac{4}{15} g_{1}{ }^{2} M_{1}^{2} \theta_{\tilde{B}}+\frac{1}{5} g_{1}^{2} S \\
& \left.+2 Y_{b}^{2}\left[m_{\tilde{D}}^{2} \theta_{\tilde{H}_{1}}+m_{\tilde{Q}}^{2} \theta_{\tilde{Q}}+m_{1}^{2} \theta_{H_{1}}+A_{b}^{2} \theta_{H_{1} \tilde{Q}}+\mu^{2}\left(\theta_{H_{2} \tilde{Q}}-2 \theta_{\tilde{H}_{1}}\right)\right]\right\} \\
& \frac{d m_{\tilde{L}}^{2}}{d t}=\frac{1}{(4 \pi)^{2}}\left\{-\left[\frac{3}{2} g_{2}^{2}\left(\theta_{\tilde{L}}-\theta_{\tilde{W}}\right)+\frac{3}{10} g_{1}^{2}\left(\theta_{\tilde{L}}-\theta_{\tilde{B}}\right)\right] m_{\tilde{L}}^{2}\right. \\
& -3 g_{2}{ }^{2} M_{2}^{2} \theta_{\tilde{W}}-\frac{3}{5} g_{1}{ }^{2} M_{1}^{2} \theta_{\tilde{B}}-\frac{3}{10} g_{1}{ }^{2} S \\
& \left.+Y_{\tau}^{2}\left[m_{\tilde{L}}^{2} \theta_{\tilde{H}_{1}}+m_{\tilde{E}}^{2} \theta_{\tilde{E}}+m_{1}^{2} \theta_{H_{1}}+A_{\tau}^{2} \theta_{H_{1} \tilde{E}}+\mu^{2}\left(\theta_{H_{2} \tilde{E}}-2 \theta_{\tilde{H}_{1}}\right)\right]\right\}
\end{aligned}
$$

${ }^{2}$ The RGEs for the soft masses of the squarks and sleptons presented here refer to the third generation of fermions. For the first two generations the Yukawa couplings should be set to zero 


$$
\begin{aligned}
& \frac{d m_{\tilde{E}}^{2}}{d t}=\frac{1}{(4 \pi)^{2}}\left\{-\left[\frac{6}{5} g_{1}^{2}\left(\theta_{\tilde{E}}-\theta_{\tilde{B}}\right)\right] m_{\tilde{E}}^{2}\right. \\
& -\frac{12}{5} g_{1}^{2} M_{1}^{2} \theta_{\tilde{B}}+\frac{3}{5} g_{1}^{2} S \\
& \left.+2 Y_{\tau}^{2}\left[m_{\tilde{E}}^{2} \theta_{\tilde{H}_{1}}+m_{\tilde{L}}^{2} \theta_{\tilde{L}}+m_{1}^{2} \theta_{H_{1}}+A_{\tau}^{2} \theta_{H_{1} \tilde{L}}+\mu^{2}\left(\theta_{H_{2} \tilde{L}}-2 \theta_{\tilde{H}_{1}}\right)\right]\right\} \\
& \frac{d m_{1}^{2}}{d t}=\frac{1}{(4 \pi)^{2}}\left\{-\left[\frac{3}{2} g_{2}{ }^{2}\left(\theta_{H_{1}}-\theta_{\tilde{H}_{1} \tilde{W}}\right)+\frac{3}{10} g_{1}{ }^{2}\left(\theta_{H_{1}}-\theta_{\tilde{H}_{1} \tilde{B}}\right)\right] m_{1}^{2}\right. \\
& -3 g_{2}{ }^{2}\left(M_{2}^{2}+\mu^{2}\right) \theta_{\tilde{H}_{1} \tilde{W}}-\frac{3}{5} g_{1}{ }^{2}\left(M_{1}^{2}+\mu^{2}\right) \theta_{\tilde{H}_{1} \tilde{B}}-\frac{3}{10} g_{1}{ }^{2} S \\
& +Y_{\tau}^{2}\left[m_{1}^{2}+m_{\tilde{L}}^{2} \theta_{\tilde{L}}+m_{\tilde{E}}^{2} \theta_{\tilde{E}}+A_{\tau}^{2} \theta_{\tilde{L} \tilde{E}}\right] \\
& \left.+3 Y_{b}^{2}\left[m_{1}^{2}+m_{\tilde{Q}}^{2} \theta_{\tilde{Q}}+m_{\tilde{D}}^{2} \theta_{\tilde{D}}+A_{b}^{2} \theta_{\tilde{Q} \tilde{D}}\right]+3 Y_{t}^{2} \mu^{2} \theta_{\tilde{Q} \tilde{U}}\right\} \\
& \frac{d m_{2}^{2}}{d t}=\frac{1}{(4 \pi)^{2}}\left\{-\left[\frac{3}{2} g_{2}{ }^{2}\left(\theta_{H_{2}}-\theta_{\tilde{H}_{2} \tilde{W}}\right)+\frac{3}{10} g_{1}{ }^{2}\left(\theta_{H_{2}}-\theta_{\tilde{H}_{2} \tilde{B}}\right)\right] m_{2}^{2}\right. \\
& -3 g_{2}{ }^{2}\left(M_{2}^{2}+\mu^{2}\right) \theta_{\tilde{H}_{2} \tilde{W}}-\frac{3}{5} g_{1}{ }^{2}\left(M_{1}^{2}+\mu^{2}\right) \theta_{\tilde{H}_{2} \tilde{B}}+\frac{3}{10} g_{1}{ }^{2} S \\
& +3 Y_{t}^{2}\left[m_{2}^{2}+m_{\tilde{Q}}^{2} \theta_{\tilde{Q}}+m_{\tilde{U}}^{2} \theta_{\tilde{U}}+A_{t}^{2} \theta_{\tilde{Q} \tilde{U}}\right] \\
& \left.+3 Y_{b}^{2} \mu^{2} \theta_{\tilde{Q} \tilde{D}}+Y_{\tau}^{2} \mu^{2} \theta_{\tilde{E} \tilde{L}}\right\}
\end{aligned}
$$

The quantity $S$ appearing in the equations above is defined as

$$
S \equiv \operatorname{Tr}\left\{\frac{Y}{2} \theta_{m} m^{2}\right\}
$$

This vanishes if universal boundary conditions are assumed for all soft scalar masses involved at the unification scale, as long as we are above all particle thresholds. This is due to the fact that $S$ is multiplicatively renormalized.

The RGEs given above refer to the third generation . For the first two generations we have just to make the appropriate replacements for the Yukawa couplings which, due to their smallness, we have assumed zero.

For the Higgs and Higgsino mixing parameters $m_{3}^{2} \equiv B \mu$ and $\mu$ respectively we have,

$$
\frac{d m_{3}^{2}}{d t}=\frac{1}{(4 \pi)^{2}}\left\{\left[-\frac{3}{4} g_{2}^{2}\left(\theta_{H_{1}}+\theta_{H_{2}}+2 \theta_{H_{1} H_{2}}-\theta_{\tilde{H}_{1} \tilde{W}}-\theta_{\tilde{H}_{2} \tilde{W}}\right)\right.\right.
$$




$$
\begin{aligned}
& -\frac{3}{20} g_{1}^{2}\left(\theta_{H_{1}}+\theta_{H_{2}}+2 \theta_{H_{1} H_{2}}-\theta_{\tilde{H}_{1} \tilde{B}}-\theta_{\tilde{H}_{2} \tilde{B}}\right) \\
& \left.+\frac{3}{2} Y_{t}^{2}+\frac{3}{2} Y_{b}^{2}+\frac{1}{2} Y_{\tau}^{2}\right] m_{3}^{2} \\
& +\mu\left[-3 g_{2}^{2} M_{2} \theta_{\tilde{H}_{1} \tilde{H}_{2} \tilde{W}}-\frac{3}{5} g_{1}^{2} M_{1} \theta_{\tilde{H}_{1} \tilde{H}_{2} \tilde{B}}\right. \\
& \left.\left.+3 A_{t} Y_{t}^{2} \theta_{\tilde{Q} \tilde{U}}+3 A_{b} Y_{b}^{2} \theta_{\tilde{Q} \tilde{D}}+A_{\tau} Y_{\tau}^{2} \theta_{\tilde{L} \tilde{E}}\right]\right\} \\
\frac{d \mu}{d t}= & \frac{1}{(4 \pi)^{2}}\left\{\frac{3}{8} g_{2}^{2}\left(\theta_{\tilde{H}_{1}}+\theta_{\tilde{H}_{2}}-8 \theta_{\tilde{H}_{1} \tilde{H}_{2}}+\theta_{H_{1} \tilde{W}}+\theta_{H_{2} \tilde{W}}\right)\right. \\
+ & \frac{3}{40} g_{1}^{2}\left(\theta_{\tilde{H}_{1}}+\theta_{\tilde{H}_{2}}-8 \theta_{\tilde{H}_{1} \tilde{H}_{2}}+\theta_{H_{1} \tilde{B}}+\theta_{H_{2} \tilde{B}}\right) \\
+ & \left.\frac{3}{4} Y_{b}^{2}\left(\theta_{\tilde{Q}}+\theta_{\tilde{D}}\right)+\frac{3}{4} Y_{t}^{2}\left(\theta_{\tilde{Q}}+\theta_{\tilde{U}}\right)+\frac{1}{4} Y_{\tau}^{2}\left(\theta_{\tilde{L}}+\theta_{\tilde{E}}\right)\right\} \mu
\end{aligned}
$$

Finally the beta functions for the three gaugino masses are

$$
\frac{d M_{i}}{d t}=S_{i} \frac{b_{i}}{(4 \pi)^{2}} g_{i}^{2} M_{i}, \quad i=1,2,3
$$

where $b_{i}$ are the beta function coefficients of the gauge couplings above all particle thresholds, i.e

$$
b_{1,2,3}=\frac{33}{5}, 1,-3
$$

and $S_{i}$ are threshold function coefficients given by,

$$
\begin{aligned}
& S_{3}=-3 \theta_{\tilde{G}}-\frac{1}{6} \sum_{i=1}^{N_{g}}\left(2 \theta_{\tilde{Q}_{i}}+\theta_{\tilde{U}_{i}}+\theta_{\tilde{D}_{i}}\right) \\
& S_{2}=-6 \theta_{\tilde{W}}-\frac{1}{2} \sum_{i=1}^{N_{g}}\left(3 \theta_{\tilde{Q}_{i}}+\theta_{\tilde{L}_{i}}\right)-\frac{1}{2}\left(\theta_{H_{1} \tilde{H}_{1}}+\theta_{H_{2} \tilde{H}_{2}}\right) \\
& S_{1}=\frac{1}{11}\left[\sum_{i=1}^{N_{g}}\left(\frac{1}{6} \theta_{\tilde{Q}_{i}}+\frac{4}{3} \theta_{\tilde{U}_{i}}+\frac{1}{3} \theta_{\tilde{D}_{i}}+\frac{1}{2} \theta_{\tilde{L}_{i}}+\theta_{\tilde{E}_{i}}\right)+\frac{1}{2}\left(\theta_{H_{1} \tilde{H}_{1}}+\theta_{H_{2} \tilde{H}_{2}}\right)\right]
\end{aligned}
$$

Electroweak symmetry breaking effects, among which especially those expressed directly through $m_{t}$ might play a role, have not been included above. Note that the threshold effects computed above at the one loop level are expected to be of the same order of magnitude as the standard 2-loop contributions to the RGE's 17 . Work on these subjects is in progress. 


\section{Acknoweledgments}

We both thank the CERN Theory Division for hospitality during a short visit in which part of this work was completed. K.T. also acknowledges illuminating conversations with C. Savoy and I. Antoniadis during a visit at Saclay in the framework of the EEC Human Capital and Mobility Network "Flavourdynamics" (CHRX-CT93-0132). A.B.L. acknoweledges support by the EEC Science Program SC1-CT92-0792.

\section{References}

[1] For reviews see:

H. P. Nilles , Phys. Rep. 110(1984)1 ;

H. E. Haber and G. L. Kane, Phys. Rep. 117(1985)75 ;

A.B.Lahanas and D.V.Nanopoulos, Phys. Rep. 145(1987) .

[2] L. E. Ibañez and G. G. Ross, Phys. Lett. 110(1982)215;

K. Inoue, A. Kakuto, H. Komatsu and S. Takeshita, Progr. Theor. Phys. 68(1982)927;71(1984)96 ;

J. Ellis, D.V. Nanopoulos and K. Tamvakis, Phys. Lett. B121(1983)123;

L. E. Ibañez, Nucl. Phys. B218(1983)514 ;

L. Alvarez-Gaumé, J. Polchinski and M. Wise, Nucl. Phys. B221(1983)495;

J. Ellis, J.S. Hagelin, D.V. Nanopoulos and K. Tamvakis, Phys. Lett. B125(1983)275.

L. Alvarez-Gaumé, M. Claudson and M. Wise, Nucl. Phys. B207(1982)96;

C. Kounnas, A.B. Lahanas, D.V. Nanopoulos and M. Quiros, phys. Lett. B132(1983)95 ; Nucl. Phys. B236(1984)438

L. E. Ibañez and C. E. Lopez, Phys. Lett. B126(1983)54 ; Nucl. Phys. B233(1984)511.

[3] G. G. Ross and R. G. Roberts, Nucl. Phys. B377(1992)571;

P. Nath and R. Arnowitt, Phys. Lett. B287(1992)89;

S. Kelley, J. Lopez, M. Pois, D. V. Nanopoulos and K. Yuan, Phys. Lett. B273(1991)423 ; Nucl. Phys. B398(1993)3;

M Olechowski and S. Pokorski, Nucl. Phys. B404(1993)590;

M. Carena, S. Pokorski and C. E. Wagner, Nucl. Phys. B406(1993)59;

M. Drees and M. M. Nojiri, Phys. Rev. D45(1992)2482;

D. J. Castaño, E.J. Piard and P. Ramond, Phys. Rev. D49(1994)4882;

P. Chankowski, Phys. Rev. D41(1990)2873 . 
[4] S. Weinberg, Phys. Lett. B91(1980)51;

C. H. Llewellyn Smith, G. G. Ross and J. Weather, Nucl. Phys B177(1981)263;

L. Hall, Nucl. Phys. B178(1981)76.

[5] T. W. Appelquist and J. Carrazzone , Phys. Rev. D11(1975)2856;

[6] N. K. Falck, Z. Phys. C30(1986)247;

M. E. Machacek and M. T. Vaughn, Nucl. Phys. B222(1983)83; B236(1984)221 ; B247(1985)70;

S. P. Martin and M. T. Vaughn, NUB-3081-93TH(1993);

Y. Yamada, KEK-TH-383(1994);

I. Jack and D. R. T. Jones, LTH-334(1994).

[7] A. B. Lahanas and K. Tamvakis , "Radiative Electroweak Symmetry breaking in the MSSM and low energy thresholds", in preparation. 


\section{Table Captions}

Table I: Threshold coefficients appearing in the renormalization group equations of the gauge and Yukawa couplings. Above all thresholds these become equal to unity.

Table II: Threshold coefficients appearing in the renormalization group equations of the trilinear scalara couplings. Above all thresholds these are vanishing. 


\begin{tabular}{|l|}
\hline \multicolumn{1}{c}{ TABLE I } \\
\hline$T_{1}=\frac{1}{33}\left[20+\theta_{\tilde{H}_{1}}+\theta_{\tilde{H}_{2}}+\frac{1}{2}\left(\theta_{H_{1}}+\theta_{H_{2}}\right)+\sum_{i=1}^{3}\left(\frac{1}{2} \theta_{\tilde{L}_{i}}+\theta_{\tilde{E}_{i}}+\frac{1}{6} \theta_{\tilde{Q}_{i}}+\frac{4}{3} \theta_{\tilde{U}_{i}}+\frac{1}{3} \theta_{\tilde{D}_{i}}\right)\right]$ \\
$T_{2}=-\frac{10}{3}+\frac{4}{3} \theta_{\tilde{W}}+\frac{1}{3}\left(\theta_{\tilde{H}_{1}}+\theta_{\tilde{H}_{2}}\right)+\frac{1}{6}\left(\theta_{H_{1}}+\theta_{H_{2}}\right)+\frac{1}{6} \sum_{i=1}^{3}\left(3 \theta_{\tilde{Q}_{i}}+\theta_{\tilde{L}_{i}}\right)$ \\
$T_{3}=\frac{7}{3}-\frac{2}{3} \theta_{\tilde{G}}-\frac{1}{18} \sum_{i=1}^{3}\left(2 \theta_{\tilde{Q}_{i}}+\theta_{\tilde{D}_{i}}+\theta_{\tilde{U}_{i}}\right)$ \\
$T_{\tau 2}=\frac{1}{4}\left[-1+4 \theta_{H_{1}}-2 \theta_{\tilde{H}_{1} \tilde{W}}-\theta_{\tilde{L} \tilde{W}}+4 \theta_{\tilde{H}_{1} \tilde{L} \tilde{W}}\right]$ \\
$T_{\tau 1}=\frac{1}{12}\left[11-4 \theta_{\tilde{B} \tilde{E}}+8 \theta_{\tilde{B} \tilde{E} \tilde{H}_{1}}-2 \theta_{\tilde{B} \tilde{H}_{1}}+4 \theta_{H_{1}}-\theta_{\tilde{B} \tilde{L}}-4 \theta_{\tilde{B} \tilde{L} \tilde{H}_{1}}\right]$ \\
$T_{\tau \tau}=\frac{1}{8}\left[2+\theta_{\tilde{H}_{1} \tilde{E}}+3 \theta_{H_{1}}+2 \theta_{\tilde{L} \tilde{H}_{1}}\right]$ \\
$T_{b 3}=\frac{1}{4}\left[6-\theta_{\tilde{G} \tilde{D}}-\theta_{\tilde{G} \tilde{Q}}\right]$ \\
$T_{b 2}=\frac{1}{4}\left[-1+4 \theta_{H_{1}}-2 \theta_{\tilde{H}_{1} \tilde{W}}-\theta_{\tilde{Q} \tilde{W}}+4 \theta_{\tilde{H}_{1} \tilde{Q} \tilde{W}}\right]$ \\
$T_{b 1}=\frac{1}{28}\left[-21-4 \theta_{\tilde{B} \tilde{D}}-18 \theta_{\tilde{B} \tilde{H}_{1}}+24 \theta_{\tilde{H}_{1} \tilde{D} \tilde{B}}+36 \theta_{H_{1}}-\theta_{\tilde{B} \tilde{Q}}+12 \theta_{\tilde{H}_{1} \tilde{Q} \tilde{B}}\right]$ \\
$T_{b t}=\frac{1}{2}\left[\theta_{H_{2}}+\theta_{\tilde{H}_{2} \tilde{U}}\right]$ \\
$T_{b b}=\frac{1}{12}\left[6+\theta_{\tilde{D} \tilde{H}_{1}}+3 \theta_{H_{1}}+2 \theta_{\tilde{Q} \tilde{H}_{1}}\right]$ \\
$T_{t 3}=\frac{1}{4}\left[6-\theta_{\tilde{G} \tilde{Q}}-\theta_{\tilde{G} \tilde{U}}\right]$ \\
$T_{t 2}=\frac{1}{4}\left[-1+4 \theta_{H_{2}}-2 \theta_{\tilde{H}_{2} \tilde{W}}-\theta_{\tilde{Q} \tilde{W}}+4 \theta_{\tilde{H}_{2} \tilde{Q} \tilde{W}}\right]$ \\
$T_{t 1}=\frac{1}{52}\left[15-18 \theta_{\tilde{H}_{2} \tilde{B}}+36 \theta_{H_{2}}-\theta_{\tilde{B} \tilde{Q}}-12 \theta_{\tilde{B} \tilde{Q} \tilde{H}_{2}}-16 \theta_{\tilde{B} \tilde{U}}+48 \theta_{\tilde{B} \tilde{U} \tilde{H}_{2}}\right]$ \\
$T_{t t}=\frac{1}{12}\left[6+3 \theta_{H_{2}}+2 \theta_{\tilde{H}_{2} \tilde{Q}}+\theta_{\tilde{H}_{2} \tilde{U}}\right]$ \\
$T_{t b}=\frac{1}{2}\left[\theta_{H_{1}}+\theta_{\tilde{H}_{1} \tilde{D}}\right]$ \\
\end{tabular}




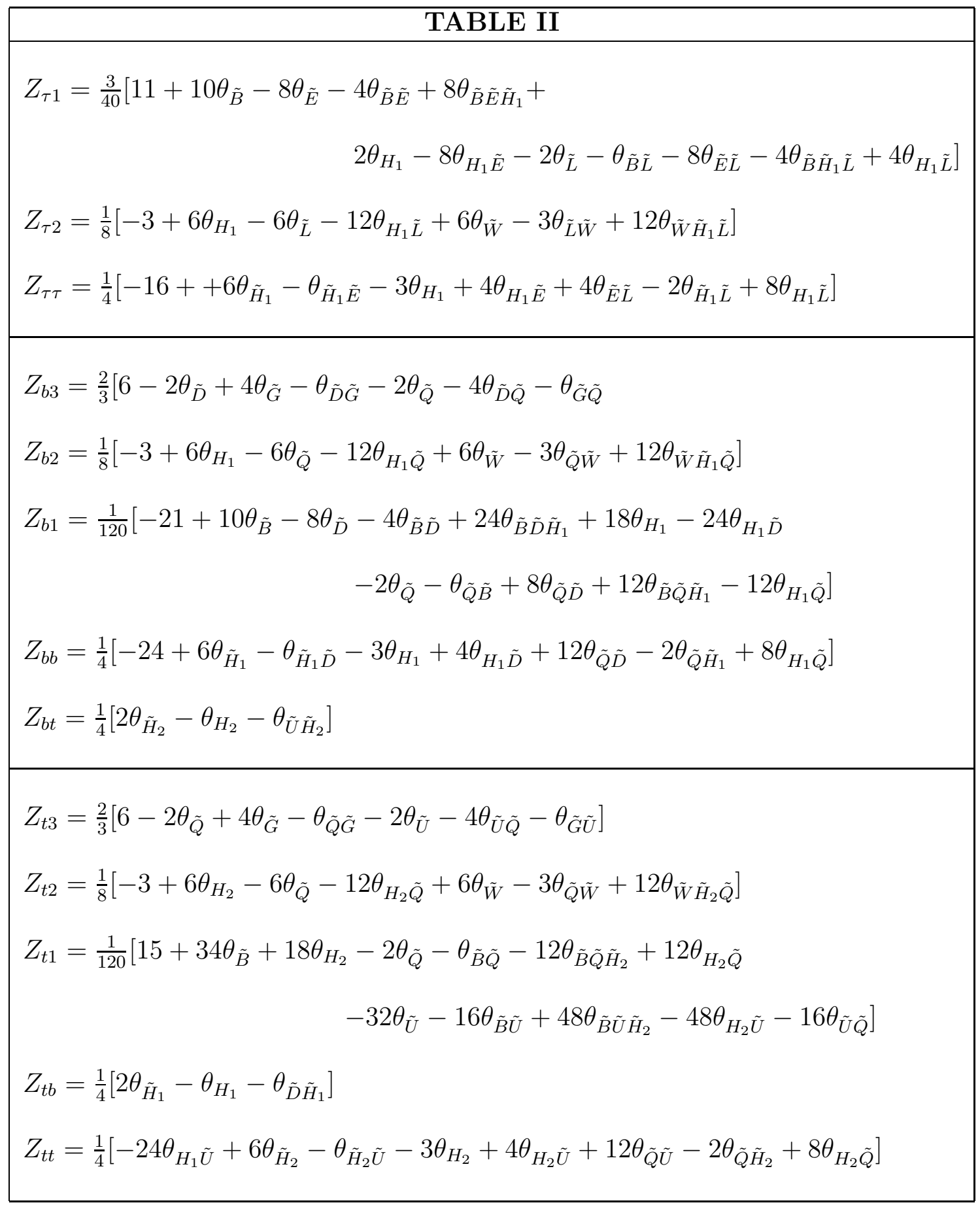

